\title{
Induced Pluripotent Stem Cells from Human Hair Follicle Mesenchymal Stem Cells
}

\author{
Yimei Wang • Jinyu Liu • Xiaohua Tan • Gaofeng Li • \\ Yunhe Gao • Xuejuan Liu • Lihong Zhang • Yulin Li \\ Published online: 16 December 2012 \\ (C) The Author(s) 2012. This article is published with open access at Springerlink.com
}

\begin{abstract}
Reprogramming of somatic cells into inducible pluripotent stem cells (iPSCs) provides an alternative to using embryonic stem cells (ESCs). Mesenchymal stem cells derived from human hair follicles (hHF-MSCs) are easily accessible, reproducible by direct plucking of human hairs. Whether these hHF-MSCs can be reprogrammed has not been previously reported. Here we report the generation of iPSCs from hHF-MSCs obtained by plucking several hairs. hHF-MSCs were isolated from hair follicle tissues and their mesenchymal nature confirmed by detecting cell surface antigens and multilineage differentiation potential towards adipocytes and osteoblasts. They were then reprogrammed into iPSCs by lentiviral transduction with Oct4, Sox2, c-Myc and Klf4. hHF-MSC-derived iPSCs appeared indistinguishable from human embryonic stem cells (hESCs) in colony morphology, expression of alkaline phosphotase, and expression of specific hESCs surface markers, SSEA-3, SSEA-4, Tra-1-60, Tra-1-81, Nanog, Oct4, ECadherin and endogenous pluripotent genes. When injected into immunocompromised mice, hHF-MSC-derived iPSCs formed teratomas containing representatives of all three germ layers. This is the first study to report reprogramming of hHF-MSCs into iPSCs.
\end{abstract}

Keywords Induced pluripotent stem cells $\cdot$ Mesenchymal stem cells $\cdot$ Human hair follicle $\cdot$ Reprogrammed

Electronic supplementary material The online version of this article (doi:10.1007/s12015-012-9420-5) contains supplementary material, which is available to authorized users.

Y. Wang $\cdot$ J. Liu $\cdot$ X. Tan $\cdot$ Y. Gao $\cdot$ X. Liu $\cdot$ L. Zhang $\cdot$ Y. Li $(\triangle)$

The Key Laboratory of Pathobiology, Ministry of Education, Norman Bethune College of Medicine, Jilin University,

Changchun 130021, People's Republic of China

e-mail: ylli@jlu.edu.cn

G. Li

Department of Spinal Surgery, Jilin Provincial People's Hospital,

Changchun, People's Republic of China

\author{
Abbreviations \\ iPSCs Induced pluripotent stem cells \\ ESCs Embryonic stem cells \\ hESCs Human embryonic stem cells \\ MSCs Mesenchymal stem cells \\ hHF-MSCs Human hair follicle mesenchymal stem cells \\ MEFs Mouse embryonic fibroblasts
}

\section{Introduction}

A major goal of stem cell research is the use of cells obtained from patient-derived pluripotent cells for therapeutic purposes. Embryonic stem cells (ESCs) are the most primitive stem cells. They are derived from preimplantation embryos, and can differentiate into all three embryonic germ layers [1]. The characterization of ESCs as totipotent has made them a powerful tool for regenerative medicine. However, hESC-based therapeutic research is technically challenging and further complicated by ethical and legal concerns, significantly limiting its potential. Therefore, it has been the goal of the scientific community to explore new and innovative approaches to obtain hESClike cells that do not destroy human embryos[2, 3].

Recently, iPSCs have been generated from both mouse and human fibroblasts following ectopic expression of the two transcription factors Oct4 and Sox2, combined with either c-Myc and Klf4 or Lin28 and Nanog [2-5]. These cells share many characteristics with ESCs, including the potential for pluripotent differentiation and capability for intensive proliferation in vitro. This result has not only facilitated disease research but also lays an important foundation for producing autologous cell therapies. Following this breakthrough, a variety of cells from mice, humans and other species have been used in reprogramming experiments, including fibroblasts[2-7], pancreatic $\beta$ cells [8], hepatocytes [9], hematopoietic cells $[10,11]$, neural stem 
cells [12, 13], and mesenchymal stem cells (MSCs) [14-16]. MSCs derived from different tissues demonstrated various reactions to the Yamanaka factors (Oct4, Sox2, c-Myc, Klf4) but were not easier to reprogram because they are relatively primitive compared with other differentiated cells. Simply using Yamanaka factors failed to induce MSCs from human bone marrow to produce cells with ESC-like properties. The addition of human telomerase to catalyze subunit hTERT and SV40 large T-Antigen was also required to reprogram these MSCs [14]. Reprogramming mouse and human adipose tissue-derived MSCs into iPSCs has a higher efficiency compared with that for mouse and human fibroblasts [15]. MSCs or progenitor cells derived from teeth or dental pulp can also be reprogrammed into iPSCs [16]. Nevertheless, similar problems were encountered when attempting to reprogram these cells, for example: limited sources, difficulty in obtaining them, and the reality that the sampling procedure itself may cause injury to the individual donor.The ideal target somatic cells should be safely and easily accessible, methods for their retrieval reproducible, and the harvesting procedure relatively noninvasive.

Hair follicles are renewable organs, they undergo repeated cycles of growth (anagen), regression (catagen), and rest (telogen) throughout the life of mammals [17].

It has been reported that there is an essential role for the follicular dermal papilla and its related dermal sheath cells in hair follicle development and regeneration [18]. At present, the majority of skin biology research is focused on epidermal stem cells. However, the characteristics of MSCs also can be observed in hair follicle dermal cells $[19,20]$. Our study first confirmed the existence of hair follicle derived MSCs obtained by direct plucking of human hairs. This method eliminates many problems associated with other procedures, and the resulting MSCs were found to be multipotent with potential to be directed toward differentiation into adipocytes and osteoblasts.

In this study, we sought to determine whether hHF-MSCs represent a cell type that may be reprogrammed to generate iPSCs. If so, this would provide a new source of reprogrammable target cells for research into personalized regenerative medicine.

\section{Materials and Methods}

Ethics Statement

After the approval of the study protocol by the Ethics committee of Jilin Province (China) and written informed consent, hHF-MSCs were obtained from the scalp of one of the authors by plucking the hairs.

\section{Cell Culture}

Several hairs with a full hair follicle were plucked, the root tissue cut with sterile ophthalmic scissors, and then placed into phosphate-buffered saline (PBS) containing penicillin and streptomycin. Hair follicle root tissues were rinsed three times in PBS containing penicillin and streptomycin, then placed into 96-well plates, cultured with hHF-MSCs medium: Dulbecco's modified Eagle's medium (DMEM)/Ham's F-12 medium (DMEM/F12; Invitrogen, Carlsbad, CA, USA), $10 \%$ fetal bovine serum (FBS; Hyclone, Victoria, Australia), $2 \mathrm{ng} / \mathrm{ml}$ basic fibroblast growth factor (bFGF; Invitrogen). Cells were passaged once the plate surface was covered with confluent cells. hHF-MSCs were frozen and stored in liquid nitrogen at passages $0-2$. Cells were thawed and expanded for experimentation at passages 2-3. CF-1 mouse embryonic fibroblasts (MEFs) used in this report were derived from an embryonic day 12.5 embryo pool of CF-I mice. MEFs were cultured in DMEM (Invitrogen) supplemented with $10 \%$ FBS. hESCs (X01) [21], obtained from the Xiaolei Group (Shanghai Institute of Biochemistry and Cell Biology, Shanghai Institutes for Biological Science, Chinese Academy of Sciences), and hHF-MSCderived iPSCs were maintained in irradiated CF-1 MEFs in hESCs culture medium ( $80 \%$ DMEM/F12 supplemented with $20 \%$ KnockOut serum replacement, $1 \%$ non-essential amino acids, $1 \mathrm{mML}$-glutamine, $4 \mathrm{ng} / \mathrm{ml}$ human $\mathrm{bFGF}$, $0.1 \mathrm{mM} \beta$-mercaptoethanol (all from Invitrogen). The hHF-MSC-derived iPSCs and hESCs were split with $1 \mathrm{mg} / \mathrm{ml}$ collagenase type IV (Invitrogen) for about $30 \mathrm{~min}$ at $37{ }^{\circ} \mathrm{C}$, at a ratio of $1: 6$ every $6-7$ days. Conditioned medium was collected according to $\mathrm{Xu}$ et al. [22].

Immunophenotyping of hHF-MSCs

Immunophenotyping was carried out as described previously [23]. Antibodies used were anti-CD29, anti-CD45, antiCD105, anti-HLA-DR (Biolegend, USA), anti-CD90, antiCD31, anti-CD73 (BD, USA) and anti-CD15 (Abcam, MA).

\section{Differentiation Experiments}

Adipogenic differentiation and detection was performed as previously described [23]. Osteogenic differentiation was induced by culturing cells at 80-90\% confluency in low glucose DMEM containing $10 \% \mathrm{FBS}, 10^{-8} \mu \mathrm{M}$ dexamethasone, $10 \mathrm{mM} \beta$-glycerophosphate (Sigma-Aldrich, USA), and $0.2 \mathrm{mML}$-ascorbic acid (Sigma-Aldrich, USA) for 3-4 weeks. Medium was replaced every 3 days. Mineralized bone nodules were detected using Alizarin Red S and Alkaline Phosphatase staining. Positive Alizarin Red S staining indicated calcium deposition, while Alkaline Phosphatase staining was black for bone nodules. 
Lentivirus Production and Establishment of hHF-MSCDerived iPSCs

We followed the protocol for lentiviral transduction as previously described [24]. Briefly, $3.6 \mu \mathrm{g}$ of lentivirus vector, pLVEF1 $\alpha$-CDNA-IRES-EGFP, was designed to carry one of the four transcription factor sequences encoding Oct4, Sox2, c-Myc or Klf4. $2.7 \mu \mathrm{g}$ of lentiviral vector pCMV $\Delta 8.91$ and $1.8 \mu \mathrm{g}$ of vesicular stomatitis virus G protein (VSV-G) were cotransfected into Human embryonic kidney (HEK) 293T cells (obtained from the Xiaolei Group, Shanghai Institute of Biochemistry and Cell Biology, Shanghai Institutes for Biological Science, Chinese Academy of Sciences) in T25 flask with Fugene HD transfection reagent (Roche, USA), respectively. Viral supernatants at 48 or $72 \mathrm{~h}$ post-transfection were collected for determination of viral titer. hHF-MSCs were transduced with a cocktail of lentiviruses carrying reprogramming factors at Day 0 . At $24 \mathrm{~h}$ post-transduction, cells were harvested by trypsinization and plated onto MEFs $\left(5 \times 10^{4}\right.$ cells per well $)$ in a six-well plate. The next day, medium was replaced with hESCs culture medium. On Day 25, iPS colonies were picked and plated onto new culture dishes.

\section{Immunostaining}

Immunostaining was carried out as previously described [25]. The primary antibodies used were: monoclonal anti-SSEA3 (1:200 dilution; Developmental Studies Hybridoma Bank); monoclonal anti-SSEA4 (1:400; Developmental Studies Hybridoma Bank); anti-Tra-1-60 (1:150; Chemicon, USA); antiTra-1-81 (1:150; Chemicon, USA); anti-Nanog (1:150; R\&D Systems, USA); anti-Oct4 (1:100; Santa Cruz Biotechnology, Santa Cruz, CA, USA); and anti-E-Cadherin (1:100; Becton Dickinson, USA).

\section{RNA Isolation and Quantitative Real-Time Polymerase Chain Reaction (qPCR)}

Total RNA was extracted with TRIZOL reagent (Invitrogen) and used as a template for reverse transcription. The qPCR assays were performed in a Bio-Rad iQ5 real-time PCR detection system (Bio-Rad, Hercules, CA, USA) using a SYBR Green PCR Master mix (TOBOYO). The PCR primers are listed in Supplemental Table S1. Each assay was carried out in triplicate. Standard curves were acquired for both the gene of interest and the internal control, glyceraldehyde-3-phsosphate dehydrogenase (GAPDH). The CT data for the gene of interest and GAPDH were obtained from the qPCR assays then transformed into copy numbers using the standard curve. The expression value of each gene was normalized to the amount of GAPDH cDNA to calculate the relative amount of RNA present in each sample. The mRNA copy number of the gene of interest was defined as the number of copies per $10^{6}$ copies of GAPDH.

Karyotype Analysis

Karyotyping was performed at the Xiangtan Center Hospital using standard protocols for high-resolution G-banding.

Teratoma Formation

The hHF-MSC-derived iPSCs were injected intramuscularly into non-obese diabetic/severe combined immune deficient (NOD/ SCID) mice (approximately $5 \times 10^{6}$ cells per site). Two hHFMSC-derived iPSC lines at a passage greater than 10 were used to produce teratomas. Three mice were injected for each cell line. Three tumors were generated when hHF-MSC-derived iPSCs 10-1 was used. Two tumors were generated when hHF-MSCderived iPSCs 20-1 was used, (10-1 and 20-1 means the generated iPSCs as the multiplicity of infection of 10 or 20). After 8 weeks, tumors were processed for hematoxylin-eosin staining.

\section{DNA Fingerprinting Analysis}

To confirm the hHF-MSCs origins of the hHF-MSC-derived iPSCs, short tandem repeat (STR) analysis was performed by Beijing Microread Gene Technology Co., Ltd. (Beijing, China).

\section{Results}

Isolation and Characterization of hHF-MSCs

The hHF-MSCs migrated outwards from the human hair follicle root tissue and adhered to the surface of the culture plate (Fig. 1a, b). They were passaged every 3-4 days to a maximum of 12 passages without major morphological alteration. The primary and passaged cells all displayed typical fibroblast-like morphology (Fig. 1d, e, f). The hair follicle is a complex organ, and it is one of the few organs of the body that undergoes cyclic bouts of degeneration and regeneration throughout life [26]. It contains a variety of different types of cells. In the primary culture, in addition to hHF-MSCs, keratinocytes were also observed around the hair follicle (Fig. 1c), as described previously by Aasen et al. [27].

Flow cytometry $\left(2 \times 10^{5}\right.$ cells were used for each antibody) demonstrated that the majority of the hHF-MSCs expressed the MSC markers CD29, CD73, CD105, CD90 (99.8\%, $98.4 \%, 84.4 \%$ and $99.5 \%$, respectively). In contrast, hematopoietic (CD31, CD45) and keratinocyte (CK15) markers were not detected. Additionally, cell populations were negative for the major histocompatibility complex (MHC) class II (HLA-DR) antigen (Fig. 1g). 

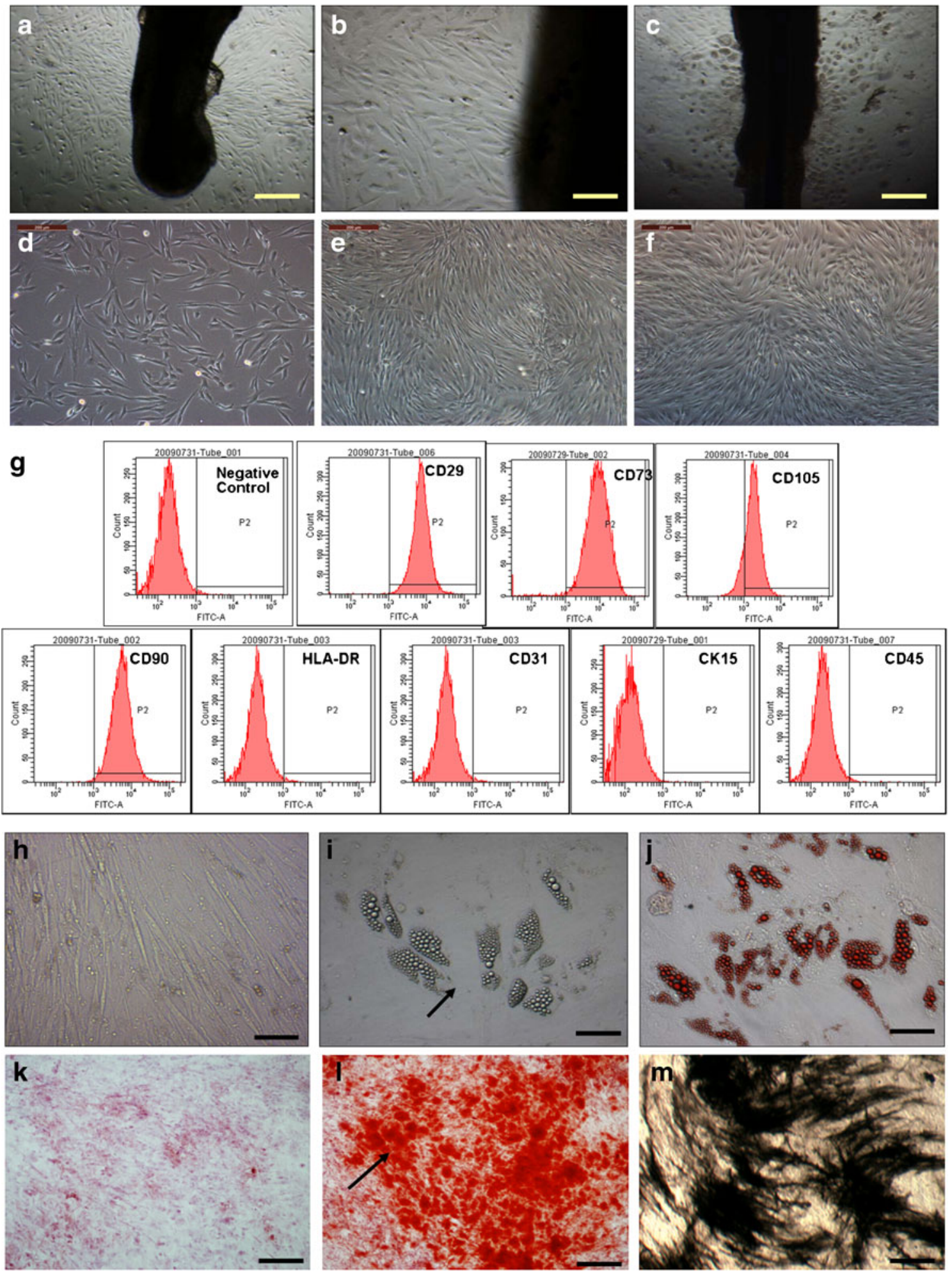

The hHF-MSCs had multilineage differentiation potential. After adipogenic induction for 4-5 days, the morphology of the cells changed from long spindle-shape into a round or polygonal shape. One week later, small bubbleshaped lipid droplets appeared in part of the cells (Supplemental Figure S1). After induction for 3 weeks, both the size and the number of lipid droplets were all increased, and most of the differentiated cells showed red lipid droplets throughout their cytoplasm (Fig. 1i, j).

When hHF-MSCs were cultured in osteogenic medium for 4 weeks, osteoblast-like cells were clearly demonstrated by alkaline phosphatase staining (Fig. 1m). In 
Fig. 1 Isolation and characterization of human hair follicle mesenchymal stem cells (hHF-MSCs). The hHF-MSCs, resembling typical fibroblast-like cells, migrated out from the hair follicles (a Bars= $500 \mu \mathrm{m}$; b Bars $=100 \mu \mathrm{m})$. Keratinocytes also migrated out from the hair follicles $(\mathbf{c}$ Bars $=500 \mu \mathrm{m})$. hHF-MSCs from passage 2 (d), passage $8(\mathrm{e})$ to passage $12(\mathrm{~F}$ Bars $=200 \mu \mathrm{m})$. Flow cytometric analysis of cell surface markers on hHF-derived fibroblast-like cells. $2 \times 10^{5}$ cells were incubated with primary antibodies against CD29, CD73, CD105, CD90, HLA-DR, CD31, CK15 or CD45, respectively, followed by incubation with a secondary FITC-labeled antibody. Controls were incubated with secondary antibody only. Percentages indicate the fraction of cells that stained positive (g). Adipogenic differentiation of hHF-MSCs. Compared to non-induced control (h Bars $=100 \mu \mathrm{m})$, induction after 3 weeks, the number of intracellular lipid droplets was further increased (i Bars $=100 \mu \mathrm{m})$ and was detected by Oil-red O staining $(\mathbf{j}$ Bars $=100 \mu \mathrm{m})$. Osteogenic differentiation of hHF-MSCs. Compared to non-induced control $(\mathbf{k}$ Bars $=200 \mu \mathrm{m})$, calcium nodules were formed after induction for 4 weeks and was demonstrated by Alizarin red staining $(\mathbf{l}$ Bars $=200 \mu \mathrm{m})$ and Alkaline phosphatase staining $(\mathbf{m}$ Bars $=200 \mu \mathrm{m})$

vitro mineralization could be shown by Alizarin red staining (Fig. 11).

\section{Formation of hESCs-Like Colonies and Their Expansion}

The schematic diagram of the reprogramming protocol is shown in Fig. 2a, and detailed methods are included in the "Materials and Methods" section. Viruses expressing a cocktail of reprogramming factors (OCT4, SOX2, C-MYC and KLF4) were used to transduce hHF-MSCs. $24 \mathrm{~h}$ after transduction, the cells were harvested by trypsinization and plated onto MEFs. Colonies with a hESCs-like morphology first became visible 7 days after transduction. When the colonies had been grown for 25-30 days, 131 colonies were picked mechanically according to the morphological characteristics of hESCs. Two hESCs-like colonies were finally selected on the basis of further characterization, and were named, hHF-MSC-derived iPSCs 10-1 and hHF-MSCderived iPSCs 20-1 (Fig. 2b).

\section{hHF-MSC-Derived iPSCs Exhibit Similar Morphology and Marker Expression to hESCs}

hHF-MSC-derived iPSCs exhibited a similar morphology to that of hESCs, characterized by a high nucleus to cytoplasm ratio and prominent nucleoli. They have now been passaged continuously for more than 20 passages ( $>120$ days). As observed in hESCs, the hHF-MSC-derived iPSCs expressed typical hESCs surface markers, including stage-specific embryonic antigen SSEA-3, SSEA-4, TRA-1-60, TRA-1-81 and E-Cadherin. They have also expressed alkaline phosphatase, Nanog (Fig. 3).

We used quantitative PCR to analyze the expression of endogenous Oct4 and Sox 2 and other pluripotency- associated genes, including Nanog, Cripto, FoxD3, Lin28, FGF4 and ESG1. Our results show that all of these genes were robustly induced. The expression of endogenous genes associated with pluripotency in hHF-MSC-derived iPSCs was effectively raised to a level similar to that observed in hESCs (Fig. 4a). These observations suggest that endogenous pluripotent genes can be fully induced by the reprogramming of hHF-MSCs. We used absolute quantitative PCR to calculate the mRNA copy number of Oct 4 and Sox 2 in these cells and found that the expression of exogenous genes Oct4 and Sox2 in hHF-MSC-derived iPSCs was downregulated compared to that in hESCs (Fig. 4b). Karyotype analysis showed that hHFMSCs at passage 5, along with hHF-MSC-derived iPSCs 10-1 at passage 10 and hHF-MSC-derived iPSCs 20-1 at passage 11 had a normal male chromosome type (46XY) with no observable chromosome abnormalities (Fig. 5). DNA fingerprinting analysis confirmed the origin of the reprogrammed colonies. (Supplemental Table S2).

\section{Teratoma Formation}

hHF-MSC-derived iPSCs were injected intramuscularly into NOD/SCID mice. At 4-5 weeks after injection we observed tumor formation. After 8 weeks, tumors had formed in three mice injected with passage $13 \mathrm{hHF}-\mathrm{MSC}$-derived iPSCs 101. Tumors also formed in two mice injected with passage 14 hHF-MSC-derived iPSCs 20-1, one of which died. Histological examination revealed that the tumors contained various tissues of the three germ layers, including pigmented epithelium (ectoderm), neural tissues (ectoderm), cartilage (mesoderm), gut-like epithelial tissues (endoderm) and respiratory epithelium (endoderm) (Fig. 6). Tumors were not observed in mice injected with hHF-MSCs only.

\section{Discussion}

It is possible to use a terminally differentiated somatic cell in order to produce a whole animal or individual via transfer of the cell nucleus or cloning $[28,29]$. Cell nucleus transfer technology has shown that differentiated cells can return to their more immature state if provided with the appropriate conditions. Recently, somatic cells have been reprogrammed directly to a pluripotent state by forced expression of four or less transcription factors to generate personalized iPSCs [2-16]. This approach not only bypasses immune rejection but also circumvents barriers of technical, ethical and legal issues which apply to the use of embryonic materials.

At present, a wide variety of cells from many different species, including humans, have been reprogrammed into iPSCs [2-16]. However, the methods for obtaining target cells for reprogramming are invasive and painful, often accompanied by restrictions on time, place and quantity of available 
a

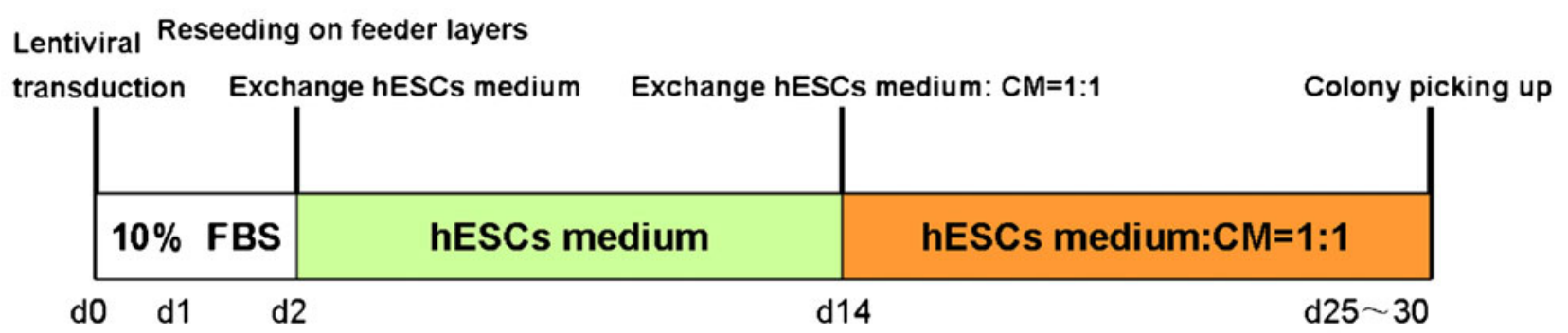
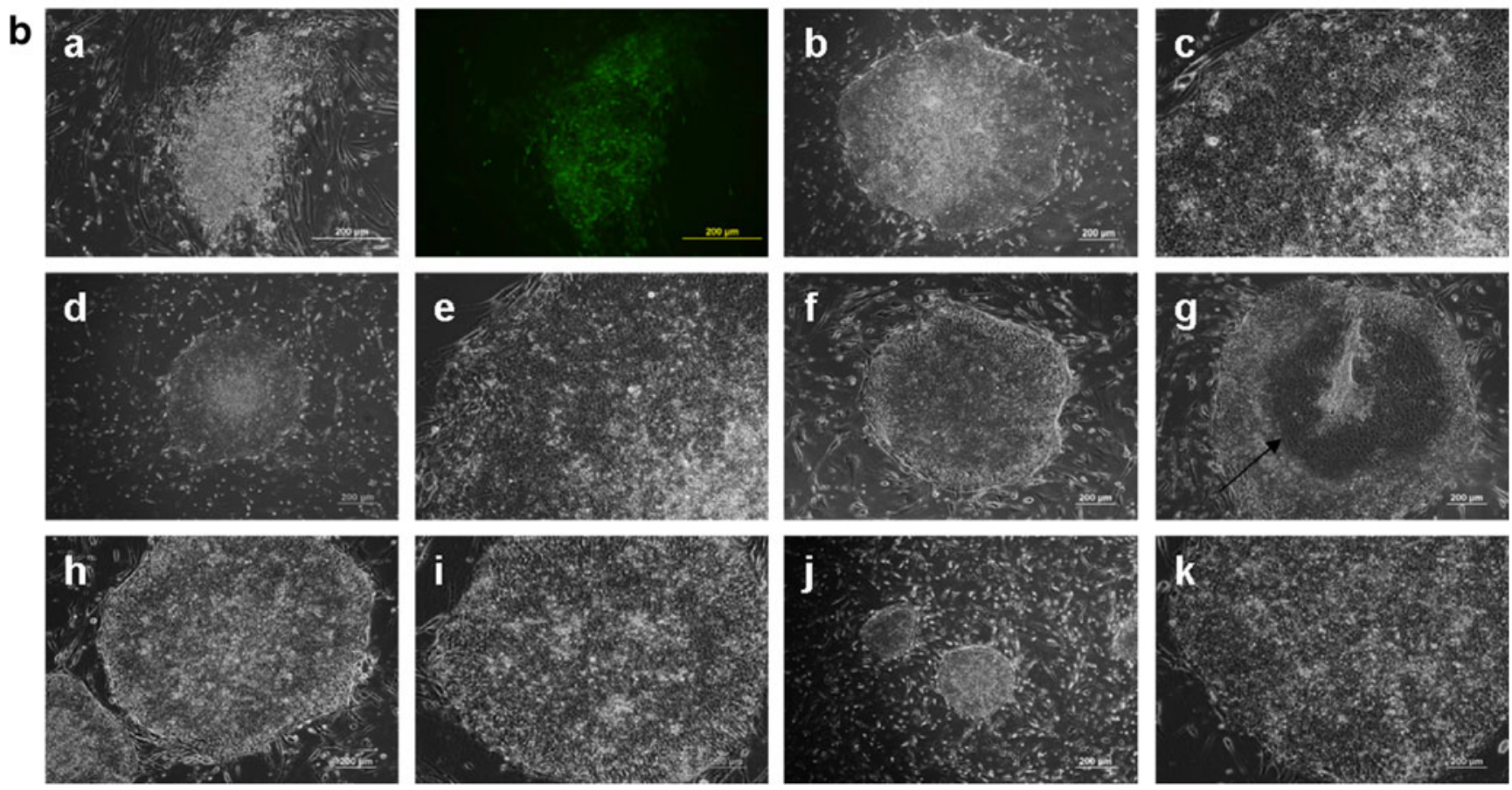

Fig. 2 Generation of induced pluripotent stem cells from hHF-MSCs. (a) Schematic diagram of the reprogramming protocol used. (b) hHFMSCs were transduced with four factors and seeded onto mouse embryonic fibroblasts until hESCs-like colonies emerged (representative colonies). (a) Typical non-hESCs-like colony. (b) hESCs colony, X01 at passage 24. (c) High magnification of the hESCs at passage 24. (d) Typical iPS colony, hHF-MSC-derived iPSCs 10-1 at passage 2. (e)

tissues. In our study we attempted to establish a simple, universally applicable (any age, sex and physical condition) and noninvasive sampling method. We also sought to determine whether hHF-MSCs represent a reprogrammable cell type for use in generating iPSCs. This is the first report to demonstrate that hHF-MSCs can be reprogrammed into iPSCs.

Multipotent MSCs were originally found in the bone marrow, and were considered to be the inherent stem cells at this location [30]. Later, these MSCs or MSC-like cells have been separated from, and identified in various tissues, including white adipose tissue, pancreas, skin, and the umbilical cord blood [31-34]. In recent years, studies have shown the presence of MSCs in the dermal sheath and dermal papillae of the hair follicle: In 2006, a comparative study investigated the properties of follicular dermal stem
High magnification of the hHF-MSC-derived iPSCs 10-1 at passage 2. (f) hHF-MSC-derived iPSCs 10-1 at passage 16. $(g$ ) Arrow indicates area of differentiation in the center of hHF-MSC-derived iPSCs 10-1 at passage 16. (h) hHF-MSC-derived iPSCs 20-1 at passage 3. (i) High magnification of hHF-MSC-derived iPSCs. at passage 3. (j) hHFMSC-derived iPSCs 20-1 at passage 17. ( $k$ ) High magnification of hHF-MSC-derived iPSCs 20-1 at passage 17. Bars $=200 \mu \mathrm{m}$

cells from whisker hairs of Wistar rats and bone marrow MSCs were isolated from femora of the same animals [35]. The results from that study showed that dermal stem cells from hair follicles have a similar morphology and population doubling time, and express the same cell-surface markers as the MSCs. Following exposure to appropriate induction stimuli, both cell populations have the capacity to differentiate into various mesenchymal lineages, such as osteoblasts, adipocytes, chondrocytes and myocytes.

Our research group tried to determine whether MSCs can be obtained from human follicles through plucking methods. Research on hair follicle stem cells indicates that most hair follicle samples have been derived from animal backskins, or the human scalp by surgical means. If we could obtain MSCs through simple plucking, it would have great 
a
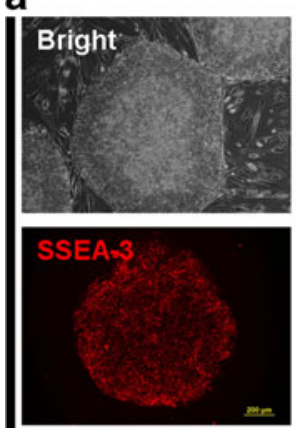

Tra-1-60
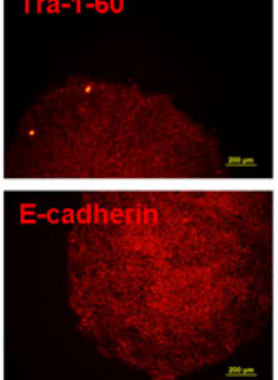
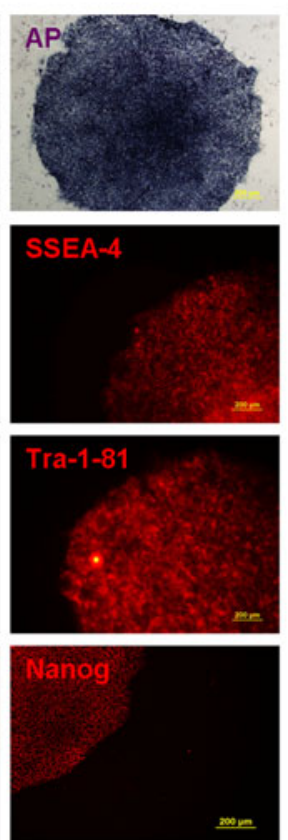

b
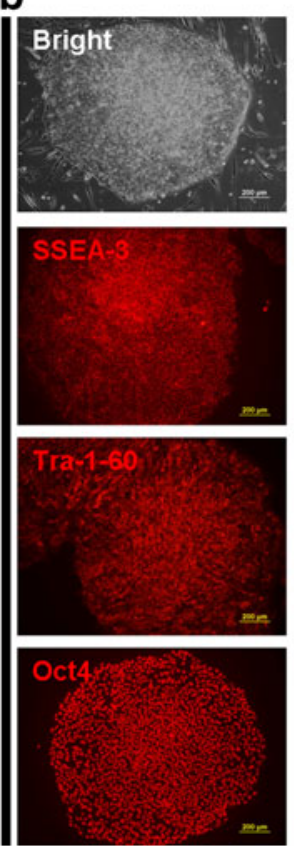
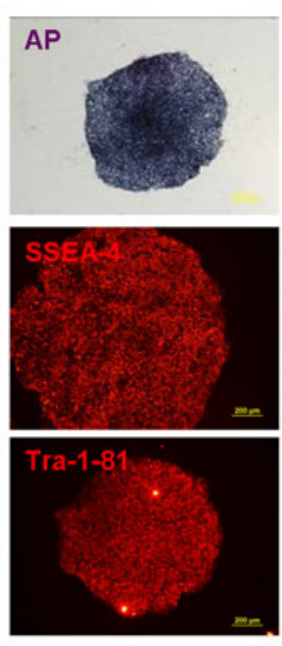

Nanog

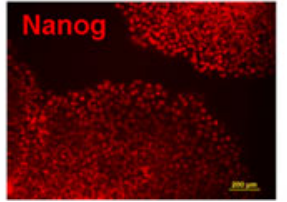

C
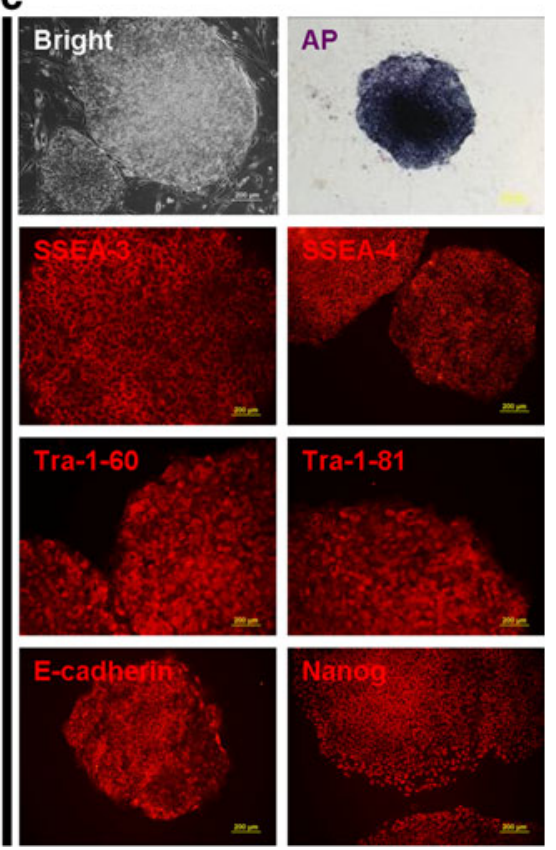

Fig. 3 hESCs pluripotency marker expression by these iPSCs. a hESCs colonies, X01 at passage 25 were fixed and stained with antibodies against hESCs associated pluripotent proteins and examined

significance for stem cell research. Recently, it has been shown that skin keratinocytes can also be obtained by the same approach and they have already been reprogrammed into iPSCs [27]. Since the specific location where hHFMSCs reside remains unclear [36], we postulate that there may be a niche in the dermal sheath and/or hair papilla. In under the fluorescence microscopy. b hHF-MSC-derived iPSCs 10-1 at passage 5. c hHF-MSC-derived iPSCs 20-1 at passage 6. Expressed proteins appeared red in the fluorescence microscope. Bars $=200 \mu \mathrm{m}$

the process of reprogramming hHF-MSCs, we observed iPS cell colony formation about 26 days after transduction, resulting in a reprogramming efficiency of $\sim 0.001 \%$. hHF-MSCs possess lower reprogramming efficiencies than do cells from human hair follicle dermal papilla which have a reprogramming efficiency of $\sim 0.02 \%$, as shown by

a
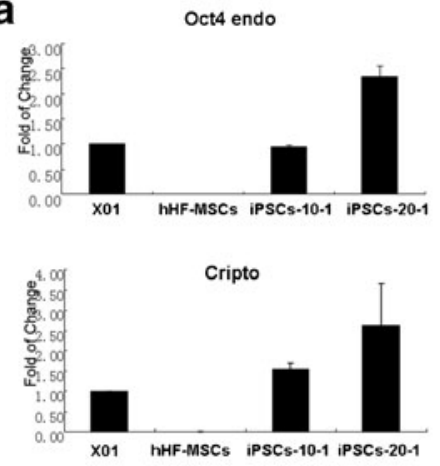

b

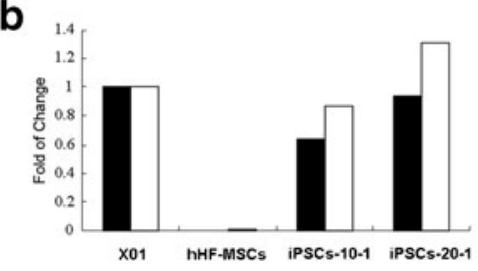

Fig. 4 a Quantitative reverse transcription-polymerase chain reaction (PCR) analyses of endogenous Oct4, Sox2, Nanog and other pluripotency genes expression in hESCs and hHF-MSC-derived iPSCs
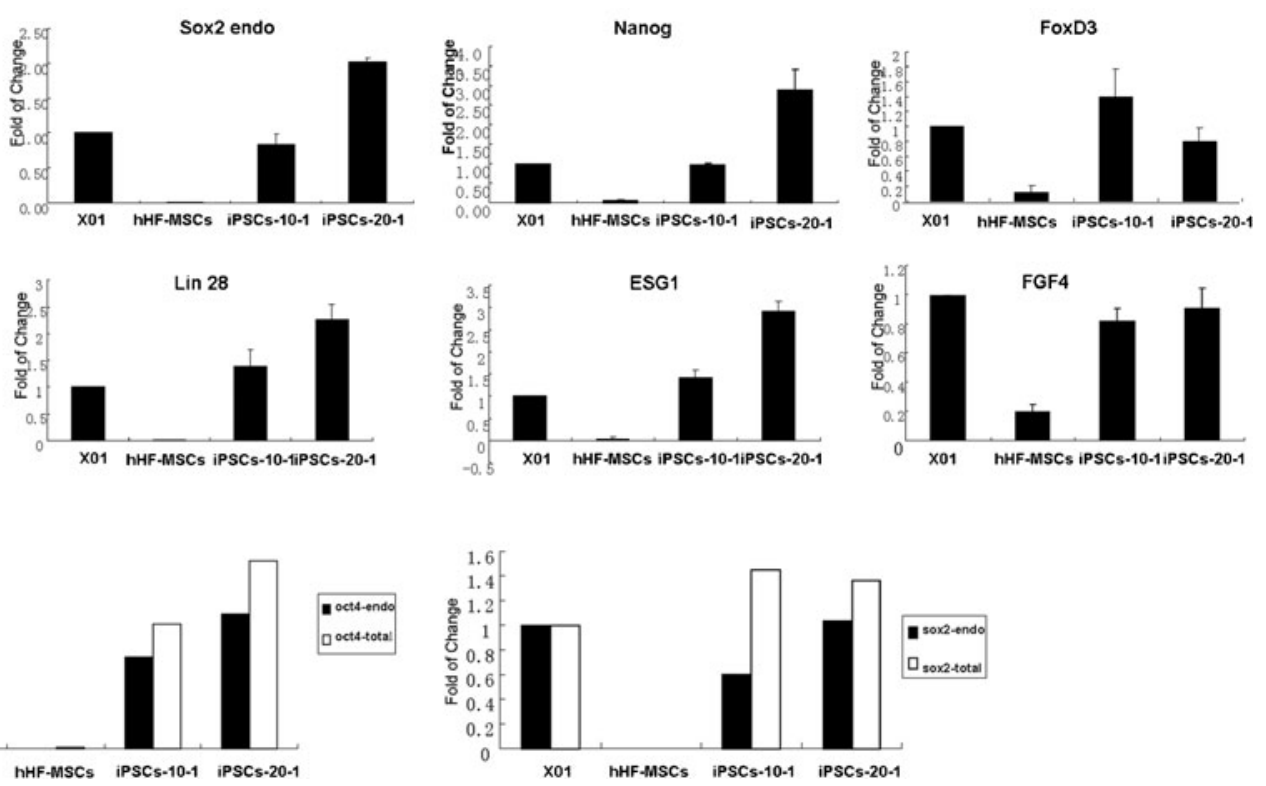

relative to parental somatic cell populations. b Quantitative RT-PCR analyses of exogenous gene Oct4 and Sox 2 expression 
a

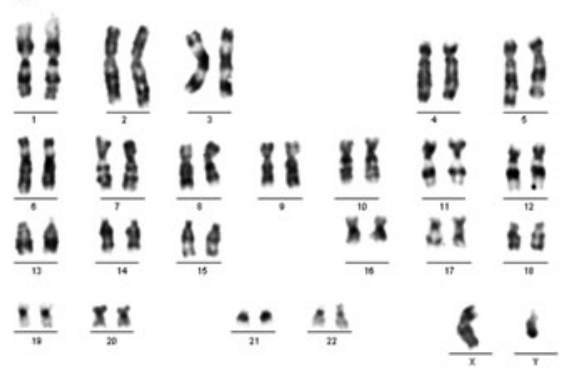

b

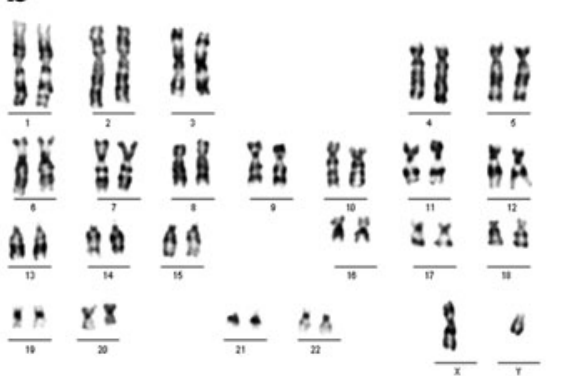

C

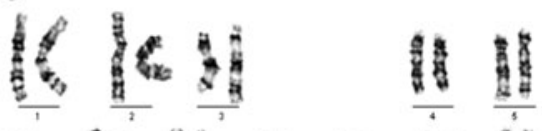

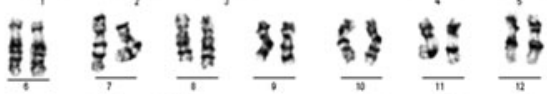

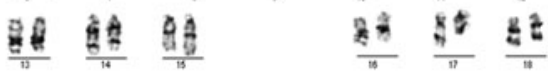

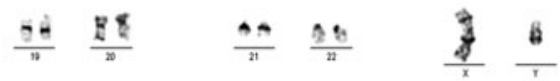

Fig. 5 Karyotype analysis. hHF-MSCs at passage 5 (a), hHF-MSC-derived iPSCs 10-1 at passage 10 (b) and hHF-MSC-derived iPSCs 20-1 at passage 11(c) all showed a normal 46XY karyotype

Christiano's research group [37]. We suggest that the possible reason for this observation is that the endogenous expression of Sox 2 and Nanog in dermal papilla cells is higher than in hHF-MSCs, which express low levels of endogenous Sox2 and Nanog (Supplemental Figure S2, Fig. 4a). Possibly these represent different cell populations of hHF-MSCs. Rendl et al., using only the transcription factors Oct 4 or Oct $4 /$ Klf4 successfully reprogrammed mouse dermal papilla cells into iPSCs $[38,39]$. This is in contrast to hHF-MSCs, which are incapable of being reprogrammed using less than four factors or use Oct 4 alone. This is the same observation as made when reprogramming human hair follicle dermal papilla cells by Christiano et al. [37]. There are other noninvasive methods to obtain cells. Pei et al. [40] have successfully derived iPSCs from exfoliated renal tubular cells present in urine samples. This maybe a preferred souce for generating iPSCs.
Although we have a relatively better way to obtain cells, the lentivirus we used for gene delivery will integrate into the genome anyway, and may cause insertional mutagenesis risks that could be resolved via usage of a different approach, such as synthetic mRNA [41] or any of the other integration-free approaches that are currently being performed in the field.

We conclude that the hHF-MSCs provides an accessible source for making iPSCs, and that hHF-MSCderived iPSCs are morphological similar to hESCs in that they exhibit a high nuclear/cytoplasmic ratio and express specific surface markers of hESCs. They also expressed pluripotency-associated transcription factor genes at similar levels as hESCs. They were able to form teratomas in vivo containing tissues of all three germ layers.
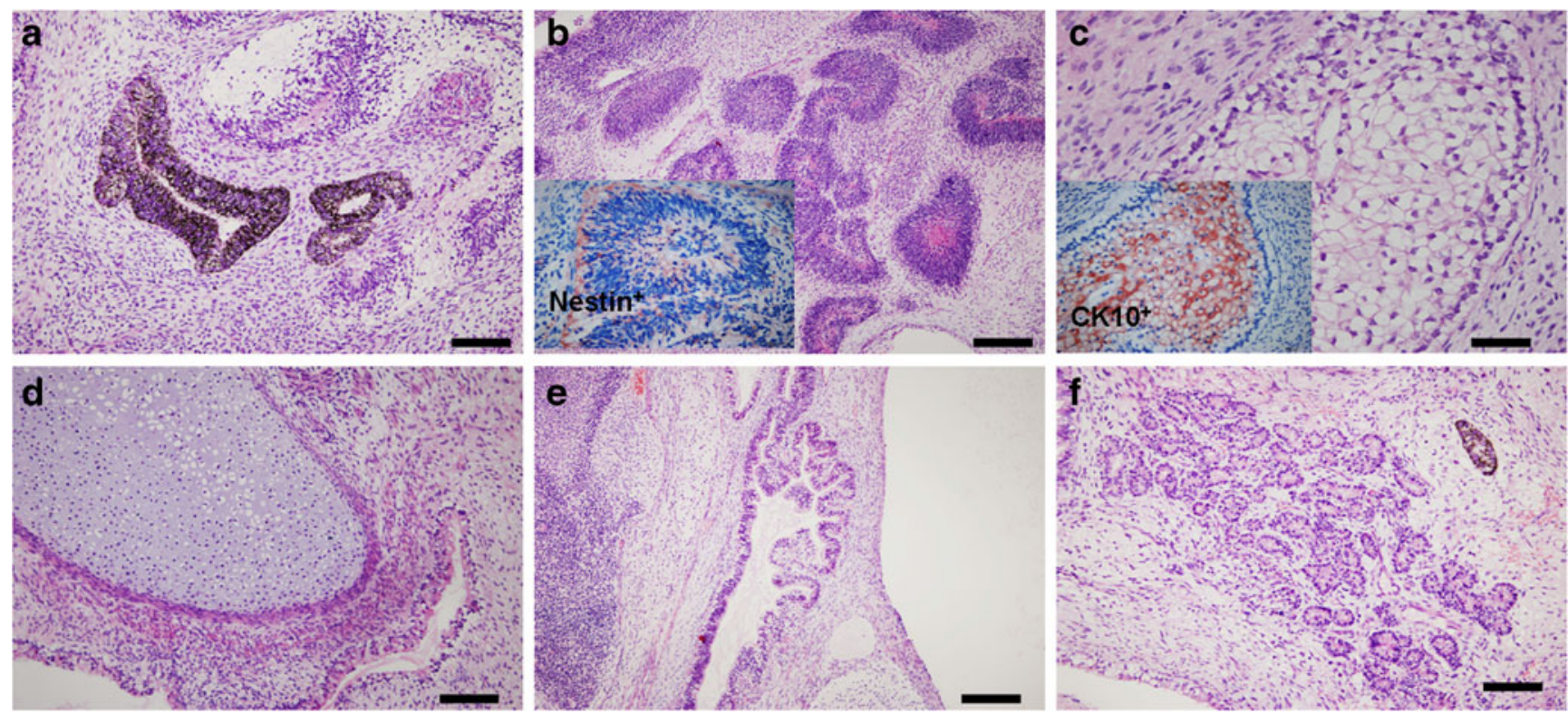

Fig. 6 Hematoxylin-eosin staining of teratomas derived from hHFMSC-derived iPSCs. Teratomas is composed of various type of tissues: (a) Pigmented epithelium (ectoderm). b Rosettes of the neural epithelium (ectoderm). c squamous epithelium (ectoderm). d cartilage tissue (mesoderm). e Respiratory epithelium (endoderm). f Gland-like structures (endoderm). Bars $=500 \mu \mathrm{m}$ 


\section{Conclusion}

In summary, MSCs were isolated from human hair follicle tissues by noninvasive means. The differentiation potential of hHF-MSCs was increased by reprogramming with Yamanaka factors. The method used to access hHF-MSCs was very convenient and enabled quick establishment of a largescale hHF-MSCs library, which was then used to create an hHF-MSC-derived iPSCs bank. This work lays the foundation for further research into how these hHF-MSC-derived iPSCs can be applied to personalized regenerative medicine.

Acknowledgments We thank Xiao Lei and Wu Zhao at the Shanghai Academy of Sciences for their technical assistance and Dr William Orr, Department of Pathology, University of Manitoba, Canada, for revising the language of this manuscript. We acknowledge the assistance given by Li Hui, Director of Reproductive Center at Xiangtan Central Hospital, for the karyotype analysis. This study was supported in part by the Major State Basic Research Development Program of China (973 Program) (2011CB606201). This work was also partially supported by National Natural Science Foundation of China (30930026/ C100101) and Science and Technology Development Plan of the Office of Science and Technology Project in Jilin Province (200905180).

Conflicts of interest The authors declare no potential conflict of interest.

Open Access This article is distributed under the terms of the Creative Commons Attribution License which permits any use, distribution, and reproduction in any medium, provided the original author(s) and the source are credited.

\section{References}

1. Thomson, J. A., Itskovitz-Eldor, J., Shapiro, S. S., Waknitz, M. A., Swiergiel, J. J., et al. (1998). Embryonic stem cell lines derived from human blastocysts. Science, 282, 1145-1147.

2. Takahashi, K., Tanabe, K., Ohnuki, M., et al. (2007). Induction of pluriptent stem cells from adult human fibroblasts by defined factors. Cell, 131, 861-872.

3. Yu, J., Vodyanik, M. A., Smuga-Otto, K., Antosiewicz-Bourget, J., Frane, J. L., et al. (2007). Induced pluripotent stem cell lines derived from human somatic cells. Science, 318, 1917-1920.

4. Wenig, M., Meissner, A., Foreman, R., et al. (2007). In vitro reprogramming of fibroblasts into a pluripotent ES-cell-like state. Nature, 448, 318-324.

5. Takahashi, K., \& Yamanaka, S. (2006). Induction of pluripotent stem cells from mouse embryonic and adult fibroblast cultures by defined factors. Cell, 126, 663-676.

6. Liu, H., Zhu, F., Yong, J., Zhang, P., Hou, P., Li, H., et al. (2008). Generation of induced pluripotent stem cells from adult rhesus monkey fibroblasts. Cell Stem Cell, 3, 587-590.

7. Esteban, M. A., Xu, J., Yang, J., Peng, M., Qin, D., Li, W., et al. (2009). Generation of induced pluripotent stem cell lines from Tibetan miniature pig. Journal of Biological Chemistry, 284, 17634-17640.

8. Stadtfeld, M., Brennand, K., \& Hochedlinger, K. (2008). Reprogramming of pancreatic beta cells into induced pluripotent stem cells. Current Biology, 18, 890-894.
9. Liu, H., Ye, Z., Kim, Y., Sharkis, S., \& Jang, Y. Y. (2010). Generation of endoderm-derived human induced pluripotent stem cells from primary hepatocytes. Hepatology, 51, 1810-1819.

10. Hanna, J., Markoulaki, S., Schorderet, P., Carey, B. W., Beard, C., et al. (2008). Direct reprogramming of terminally differentiated mature B lymphocytes to pluripotency. Cell, 133, 250-264.

11. Eminli, S., Foudi, A., Stadtfeld, M., Maherali, N., Ahfeldt, T., et al. (2009). Differentiation stage determines potential of hematopoietic cells for reprogramming into induced pluripotent stem cells. Nature Genetics, 41, 968-976.

12. Kim, J. B., Zaehres, H., Wu, G., Gentile, L., Ko, K., et al. (2008). Pluripotent stem cells induced from adult neural stem cells by reprogramming with two factors. Nature, 454, 646-650.

13. Kim, J. B., Greber, B., AraÚzo-Bravo, M. J., Meyer, J., Park, K. I., et al. (2009). Direct reprogramming of human neural stem cells by OCT4. Nature, 461, 649-653.

14. Park, I. H., Zhao, R., West, J. A., Yabuuchi, A., Huo, H., et al. (2008). Reprogramming of human somatic cells to pluripotency with defined factors. Nature, 451, 141-146.

15. Sugii, S., Kida, Y., Kawamura, T., Suzuki, J., Vassena, R., et al. (2010). Human and mouse adipose-derived cells support feeder independent induction of pluripotent stem cells. Proceedings of the National Academy of Sciences, 107, 3558-3563.

16. Yan, X., Qin, H., Qu, C., Tuan, R. S., Shi, S., et al. (2010). iPS cells reprogrammed from human mesenchymal-like stem/progenior cells of dental tissue origin. Stem Cells and Development, 19, 469-480.

17. Hoffman, R. M. (2000). The hair follicle as a gene therapy target. Nature Biotechnology, 18, 20-1.

18. Ohyama, M., Zheng, Y., Paus, R., \& Stemm, K. S. (2009). The mesenchymal component of hair follicle neogenesis: background, methods and molecular characterization. Experimental Dermatology, 19, 89-99.

19. Richardson, G. D., Arnott, E. C., Whitehouse, C. J., Lawrence, C. M., Hole, N., et al. (2005). Cultured cells from the adult human hair follicle demis can be directed toward adipogenic and osteogenic differentiation. The Journal of Investigative Dermatology, 124, 1090-1091.

20. Reynolds, A. J., Lawrence, C., Cserhalmi-Friedman, P. B., Christiano, A. M., \& Jahoda, C. A. (1999). Trans-gender induction of hair follicles. Nature, 402, 33-34.

21. Wu, Z., Li, H., Rao, L., He, L., Bao, L., et al. (2011). Derivation and characterization of human embryonic stem cell lines from the Chinese population. Journal of Genetics and Genomics, 38, 13-20.

22. Xu, C., Inokuma, M. S., Denham, J., Golds, K., Kundu, P., et al. (2001). Feeder-free growth of undifferentiated human embryonic stem cells. Nature Biotechnology, 19, 971-974.

23. Yang, X. F., He, X., He, J., Zhang, L. H., Xj, S., et al. (2011). High efficient isolation and systematic identification of human adiposederived mesenchymal stem cells. Journal of Biomedical Science, $18,59$.

24. Liao, J., Wu, Z., Wang, Y., Cheng, L., Cui, C., et al. (2008). Enhanced efficiency of generating induced pluripotent stem (iPS) cells from human somatic cells by a combination of six transcription factors. Cell Research, 18, 600-603.

25. Xiao, L., Yuan, X., \& Sharkis, S. J. (2006). Activin A maintains self-renewal and regulates fibroblast growth factor, Wnt, and bone morphogenic protein pathways in human embryonic stem cells. Stem Cells, 24, 1476-1486.

26. Fuchs, E. (2007). Scratching the surface of skin development. Nature, 445, 834-842.

27. Aasen, T., \& Izpisúa Belmonte, J. C. (2010). Isolation and cultivation of human keratinocytes from skin or plucked hair for the generation of induced pluripotent stem cells. Nature Protocols, 5, 371-382.

28. Solter, D. (2000). Mammalian cloning: advances and limitations. Nature Reviews Genetics, 1, 199-207. 
29. Rideout, W. M., Eggan, K., \& Jaenisch, R. (2001). Nuclear cloning and epigenetic reprogramming of the genome. Science, 293, 1093-1098.

30. Uccelli, A., Moretta, L., \& Pistoia, V. (2008). Mesenchymal stem cells in health and disease. Nature Reviews Immunology, 8, 726-736.

31. Zuk, P. A., Zhu, M., Ashjian, P., De Ugarte, D. A., Huang, J. I., et al. (2002). Human adipose tissue is a source of multipotent stem cells. Molecular Biology of the Cell, 13, 4279-4295.

32. Baertschiger, R. M., Bosco, D., Morel, P., Serre-Beinier, V., Berney, T., et al. (2008). Mesenchymal stem cells derived from human exocrine pancreas express transcription factors implicated in beta-cell development. Pancreas, 37, 75-84.

33. Metcalfe, A. D., \& Ferguson, M. W. (2008). Skin stem and progenitor cells: using regeneration as a tissue-engineering strategy. Cellular and Molecular Life Sciences, 65, 24-32.

34. Markov, V., Kusumi, K., Tadesse, M. G., William, D. A., Hall, D. M., et al. (2007). Identification of cord blood-derived mesenchymal stem/stromal cell populations with distinct growth kinetics, differentiation potentials, and gene expression profiles. Stem Cells and Development, 16, 53-73.

35. Hoogduijn, M. J., Gorjup, E., \& Genever, P. G. (2006). Comparative characterization of hair follicle dermal stem cells and bone marrow mesenchymal stem cells. Stem Cell and Development, 15, 49-60.
36. Toma, J. G., Akhavan, M., Fernandes, K. J., Barnabé-Heider, F., Sadikot, A., et al. (2001). Isolation of multipotent adult stem cells from the dermis of mammalian skin. Nature Cell Biology, 3, 778-784.

37. Higgins, C. A., Itoh, M., Inoue, K., Richardson, G. D., Jahoda, C. A., et al. (2012). Reprogramming of human hair follicle dermal papilla cells into induced pluripotent stem cells. The Journal of Investigative Dermatology, 132, 1725-1727.

38. Tsai, S. Y., Bouwman, B. A., Ang, Y. S., Kim, S. J., Lee, D. F., et al. (2011). Single transcription factor reprogramming of hair follicle dermal papilla cells to induced pluripotent stem cells. Stem Cells, 29, 964-971.

39. Tsai, S. Y., Clavel, C., Kim, S., Ang, Y. S., Grisanti, L., et al. (2010). Oct4 and klf4 reprogram dermal papilla cells into induced pluripotent stem cells. Stem Cells, 28, 221-228.

40. Zhou, T., Benda, C., Duzinger, S., Huang, Y., Li, X., et al. (2011). Generation of induced pluripotent stem cells from urine. Journal of the American Society of Nephrology, 22, 1221-1228.

41. Warren, L., Manos, P. D., Ahfeldt, T., Loh, Y. H., Lau, F., et al. (2010). Highly efficient reprogramming to pluripotency and directed differentiation of human cells with synthetic modified mRNA. Cell Stem Cell, 7, 618-630. 